\title{
Effect of Family History of Hypertension on Left Ventricular Mass in Normotensive Individuals
}

\author{
Yerrolla Mounica ${ }^{1}$, A. Santa Kumari ${ }^{2}$, Geetha Shavali ${ }^{3}$ \\ ${ }^{1}$ Postgraduate, ${ }^{2}$ Professor and Head of the Department, ${ }^{3}$ Assisstant Professor, \\ Upgraded Department of Physiology, Osmania Medical College, Hyderabad, Telangana
}

\begin{abstract}
Introduction: Presence of family history of hypertension is a strong predictor of development of hypertension in normotensive subjects. Increased left ventricular mass might antedate the development of hypertension in the individuals destined to develop systemic hypertension in life.
\end{abstract}

Aim: To study the left ventricular mass in offspring of normotensive parents (ONP) and offspring of hypertensive parents (OHP) and to find the variations in between the two groups.

Materials and Method: 75 healthy normotensive individuals with family history of hypertension and 75 healthy normotensive individuals without family history of hypertension were included in the study .LV mass was calculated by using 2D M-mode echocardiography by the formula given by American society of Echocardiography, LV mass $=0.8 \times[1.04 \times($ LVIDd + IVSd + PWd $) 3-($ LVIDd $) 3]+0.6$ grams. [LVIDd Left ventricular internal diameter during diastole; IVSd- Interventricular septal thickness during diastole; PWd - Posterior wall thickness during diastole].

Observation and Results: Left ventricular mass was significantly higher in the normotensive individuals with familyhistory of hypertension $(64.33 \pm 20.22 \mathrm{~g})$ when compared to those without family history $(56.12 \pm 16.13 \mathrm{~g})$.

Conclusion: The present study reveals increased left ventricular mass in normotensive individuals with family history of hypertension. Early evaluation of left ventricular mass and life style modification are recommended in healthy normotensive individuals with family history of hypertension to prevent and delay the clinical symptomatolgy of hypertension.

Keywords: Left ventricular mass, echocardiography, hypertension.

\section{Introduction}

Hypertensive heart disease is a complex entity involving cardiovascular changes due to arterial hypertension .Heart as a pump of circulatory system is effected by the increased blood pressure from the early stages of hypertension and actually suffers the common

\section{Corresponding Author:}

\section{Dr. Yerrolla Mounica}

Osmania Medical College, Mounika

e-mail: yerrolla23@gmail.com hypertension related organ damage ${ }^{8}$. Current approach to the management of systemic hypertension is early diagnosis and identification of individuals at risk through detection of early markers of the disease ${ }^{9}$. Left ventricular hypertrophy is one of the pathological hallmarks of systemic hypertension. Increased left ventricular mass might antedate the development of hypertension in the individuals destined to develop systemic hypertension in life. ${ }^{1}$ Since essential hypertension is thought to have an important genetic component in its causation, offspring of hypertensive parents represent an excellent opportunity to study the early subclinical phases of syndrome of systemic hypertension. The main purpose of the present 
study was to evaluate left ventricular structure in normotensive Offspring of hypertensive parents (OHP) with the aim of detecting early abnormalities preceding the onset of systemic hypertension .

\section{Materials and Method}

A cross sectional hospital based study including 75 normotensive individuals with family history of hypertension and 75 normotensive individuals without family history of hypertension between the age group of $30-40$ years was conducted in Osmania general hospital. Subjects with blood pressure $>130 / 90 \mathrm{mmHg}$, with family history of - Diabetes mellitus,Thyroid disorders and subjects using cardioactive drugs were excluded from the study.

\section{Materials:}

1. Automatic blood pressure monitor

2. Height measuring stand

3. Digital weighing machine

4. 2D M-mode Echocardiographic machine

Procedure: Institutional ethics committee approval and informed consent was taken.
Height, weight and average of two blood pressure recordings with a time gap of 10 min was taken . BMI was calculated using Queteletformula BMI = weight in $\mathrm{kg} /$ height in $\mathrm{m} 2$. Echocardiography was done in the partial left lateral decubitus position after a resting period of 3-4 minutes. A 2D guided M- mode recording of the left ventricle was obtained in the parasternal long axis view and measurements of wall thickness and chamber diameter were determined in diastole in accordance with method outlined by American society of echocardiography. The mean value of the parameters were taken from three consecutive beats.LVM was calculated using the formula given by American society of echocardiography

$$
\begin{aligned}
& \mathrm{LVM}=0.8 \times[1.04 \times(\mathrm{LVIDd}+\mathrm{IVSd}+\mathrm{PWd}) 3- \\
& (\mathrm{LVIDd}) 3]+0.6 \text { grams }
\end{aligned}
$$

\section{Observations and Results}

Statistical analysis was done using unpaired students $\mathrm{t}$ test and $\mathrm{P}$ value $<0.05$ was considered as statistically significant.

Table 1: Age and gender distribution in OHP and ONP

\begin{tabular}{|l|c|c|c|}
\hline & OHP & ONP & P Value \\
\hline Age & $37.32 \pm 3.81$ & $37.63 \pm 2.98$ & $0.584 \mathrm{NS}$ \\
\hline Males & $37(49.5 \%)$ & $37(49.5 \%)$ & $0.47 \mathrm{NS}$ \\
\hline Females & $38(50.5 \%)$ & $38(50.5 \%)$ & $0.47 \mathrm{NS}$ \\
\hline
\end{tabular}

Table 2: Comparison of anthropometric measurements in OHP and ONP

\begin{tabular}{|l|c|c|c|}
\hline & OHP & ONP & P Value \\
\hline Height $($ Inch) & $5.1 \pm 1.0$ & $4.9 \pm 1.3$ & $0.22 \mathrm{NS}$ \\
\hline Weight $(\mathrm{kg})$ & $64 \pm 12$ & $68 \pm 9$ & $0.61 \mathrm{NS}$ \\
\hline BMI & $34.88 \pm 8.68$ & $32.67 \pm 5.66$ & $0.03 \mathrm{~S}$ \\
\hline
\end{tabular}

Table 3: Comparison of systolic and diastolic blood pressure in OHP and ONP

\begin{tabular}{|l|c|c|c|}
\hline & OHP & ONP & P Value \\
\hline SYSTOLIC BP & $116.0 \pm 8.85$ & $118.27 \pm 8.75$ & $0.117 \mathrm{NS}$ \\
\hline DIASTOLIC BP & $75.20 \pm 7.94$ & $77.47 \pm 8.39$ & $0.092 \mathrm{NS}$ \\
\hline
\end{tabular}


Table 4: Comparison of left ventricular geometric parameters in OHP and ONP

\begin{tabular}{|l|c|c|c|}
\hline & OHP & ONP & P Value \\
\hline IVS & $1.13 \pm 0.12$ & $1.01 \pm 0.02$ & $0.12 \mathrm{NS}$ \\
\hline PWT & $0.95 \pm 0.12$ & $0.89 \pm 0.52$ & $0.47 \mathrm{NS}$ \\
\hline LVIDd & $4.29 \pm 0.32$ & $4.12 \pm 0.57$ & $0.53 \mathrm{NS}$ \\
\hline LVIDs & $2.93 \pm 0.43$ & $2.57 \pm 0.34$ & $0.32 \mathrm{NS}$ \\
\hline LVM & $126.15 \pm 28.04$ & $104.56 \pm 28.01$ & $0.01 \mathrm{~S}$ \\
\hline
\end{tabular}

\section{Discussion}

The results of this study showed that left ventricular mass was significantly higher in OHP . The presence study suggests that alterations in left ventricular structure may also have genetic basis as there is abnormal left ventricular geometry in normotensive offspring of hypertensive parents. This is supported by a recent study by Lam et al which showed familial aggregation of left ventricular geometry in a two generation communitybased sample. ${ }^{2}$.

At equal blood pressure levels, some individuals develop LV hypertrophy, whereas others do not,indicating a genetic susceptibility of the development of LV hypertrophy. Because the major causes of morbidity and mortality among hypertensive patients are due to the cardiovascular manifestations of hypertension and not the level of blood pressure per se, understanding the genetic susceptibility of the LV hypertrophy to the effects of hypertensionremains a matter of intense interest ${ }^{5}$.

Left ventricular mass (LVM) is a significant risk factor for cardiovascular disease and hence it is an important clinicalmeasure in both healthy individuals and patients with disease, LVM is determined by a combination of genetic factors, environmental andmechanical factors. During embryogenesis, heart developmentis under the control of a series of transcription and growth factors acting on cell differentiation and on heartmorphogenesis ${ }^{6}$.

Left ventricular structural adaptation is highly dependent on oxidation of glucose and fatty acids by mitochondria. A plausible working hypothesis is therefore that the close concordance between mothers and offspring might be explained by mitochondrial DNA that sons and daughters inherit from them. The mendelian hypothesis that offspring derived their genetic makeup in equal proportions from their mothers and fathers is known to be incorrect. Genomic imprinting is the differential modification of the maternal and paternal contributions to the zygote. Thus,during development and growth into adulthood offspring are under influences of distinct maternal and paternal "imprints" that result in divergent expression of parental alleles. The intrauterine environment plays a pivotal role in the development of the fetus and is predominantly determined by genetic and environmental factors linked to the mother ${ }^{7}$.

In the Dutch Hypertension and Offspring Study, the thickness of LV posterior wall and inter-ventricular septum were increased in the offspring of hypertensive parents but the difference from the offspring of normotensive parents was not significant. However, LV mass and LV end-diastolic diameter were significantly increased in the former group. This is in agreement with our study which also showed significant increase in LVM in OHP when compared to ONP.

Family history of cardiovascular diseases are predictors of cardiovascular risk in adulthood and the relationship between them starts a symptomatically from the first years of life. High blood pressure (HBP) is considered one of the major risk factors for cardiovascular disease among them. Normotensive children of hypertensive parents are exposed to greater risk of developing hypertension in adulthood as they have shown early cardiovascular changes.

There are many reports on left ventricular mass heritability with estimates from different populations. Most studies showed significant heritability and sibling correlations, especially among African Americans. The heritability decreased after matching the blood pressure. It implied possible pleiotrophic effects of genes on controlling blood pressure and LV mass. Among young adults and adolescents, Hemodynamicload, such as stroke volume, has an influence on LV mass, and this impact ismore important than body size. However, 
high proportions of LV mass variations still remain unexplained Genetic components played important role in residual LVmass variations ${ }^{4}$.

In this study the relation between LVM measured by echocardiography with BMI and blood pressure was studied which showed that there was significant increase in LVM in subjects with increased BMI. This is in agreement with the study done by Chad garner et al. which showed that the strongest predictor of LVM was BMI and they both are strongly correlated .

Such a correlation implies that at least some of the familial factors that affect weight also affect LVM. Thus, if the familial factors are genetic, this would suggest that at least some of the genes acting on the traits are pleiotropic, that is influence both traits. Thus, LVM and weight are determined in large part by common genetic and/or familial environmental factors in both adults and children, whereas the impact of correlated nonfamilial environmental factors appears to be much larger in children compared with adults ${ }^{6}$.

Left ventricular (LV) hypertrophy profoundly affects morbidity and mortality from cardiovascular diseases, including myocardial infarction, congestive heart failure, and stroke. Therefore, it is important to measure LV mass and manage LV hypertrophy among the hypertensive population in clinical practice. The understanding of natural history of LVhypertropy has been enhanced by noninvasive imaging method of echocardiography that have greatly increased the capability of determining the increased LVmass. Blood pressure and volume overload are considered as a strong determinant of LV hypertrophy. However, interindividual variations in LV mass can be explained, only to a limited extent, by hemodynamic load . Moreover, LV hypertrophy may occur in the absence of hypertension. Genetic components are considered to be important factors for LV mass, and it has been proved by twin studies which showed that heritability accounts for up to 20-70\%.Significant heritability and evidence of strong familial aggregation in LV mass have been reported in different populations, such as in Caucasian, African Americans, American Indians and Caribbean Hispanic families .Previous segregation study showed the mode of inheritance of LV mass was compatible to polygenic model, but the study did not prove major gene effects controlling LV mass. The existence of major gene effects can be investigated by segregation analysis besides the polygenic background effects, and help to facilitate the further genomic study.

Our results are contrast to that of Jalal et al who found no significant difference in LVM between the two groups. This may be because our patients were older than those evaluated by Jalal et al and hence age does appear to be an important factor in the expression of LVM phenotypes in OHP . It is possible and seems likely that their subjects might have been examined at an earlier stage in the natural history before the full expression of the morphological cardiovascular changes of the preclinical hypertension syndrome

Graettinger, et al reported the absence of any significant difference in LV mass index or wall thickness between normotensive healthy volunteers with or without genetic risk for hypertension ${ }^{5}$. However, their contention that such a lack of difference could be due to a meaningful difference in diastolic blood pressure between the two groups cannot be offered as a similar explanation for our observations since our subject groups were properly matched for the blood pressure level

\section{Conclusions}

The present study reveals:

IN OHP - 1. $\uparrow$ BMI is associated with $\uparrow \mathrm{LVM}$

2. Even with normal BMI there is $\uparrow$ LVM

IN ONP - 1. $\uparrow \mathrm{BMI}$ is associated with $\uparrow \mathrm{LVM}$

2. With normal BMI, LVM is in normal range

This clearly points that not only genetic basis but also BMI is an individual risk factor for developing cardiovascular morbidity.

Life style modifications would protect and prevent these complications.

\section{Conflict of Interest: None}

Source of Funding: Self

Ethical Clearance: Obtained

Acknowledgements: I Express my sincere gratitude to all the faculty and postgraduates of Upgraded department of physiology, Osmania medical college and to all my study population.

\section{References}

1. Jalal S, Rauoof MA, Khan KA, Hamid S, Waheed 
A, Jan VM, Lone NA, Rather HA, Habib K, Alai SM, Kumar D. Left ventricular mass and functions in normotensive offspring of hypertensive parents: an echocardiographic study. J Assoc Physicians India. 2009 May;57:389-92.

2. Lam CS, Liu X, Yang Q, Larson MG, Pencina MJ, Aragam J, Redfield MM, Benjamin EJ, Vasan RS. Familial aggregation of left ventricular geometry and association with parental heart failure: the Framingham Heart Study. Circulation: Cardiovascular Genetics. 2010 Dec;3(6):492-8.

3. Graettinger WF, Neutel JM, Smith DH, Weber MA. Left ventricular diastolic filling alterations in normotensive young adults with a family history of systemic hypertension. The American journal of cardiology. 1991 Jul 1;68(1):51-6.

4. Chien KL, Hsu HC, Su TC, Chen MF, Lee YT. Heritability and major gene effects on left ventricular mass in the Chinese population: a family study. BMC cardiovascular disorders. 2006 Dec 1;6(1):37.
5. Arnett DK. Genetic contributions to left ventricular hypertrophy. Current hypertension reports. 2000 Feb 1;2(1):50-5.

6. Garner C, Lecomte E, Visvikis S, Abergel E, Lathrop M, Soubrier F. Genetic and environmental influences on left ventricular mass: a family study. Hypertension. 2000 Nov;36(5):740-6.

7. Kuznetsova T, Staessen JA, Olszanecka A, Ryabikov A, Stolarz K, Malyutina S, Fagard R, Kawecka-Jaszcz K, Nikitin Y. Maternal and paternal influences on left ventricular mass of offspring. Hypertension. 2003 Jan 1;41(1):69-74.

8. Karabinos I, Grassos C, Kostaki P, Kranidis A. Echocardiography in the evaluation of a hypertensive patient: an invaluable tool or simply following the routine. Hellenic J Cardiol. 2013 Jan 1;54:47-57.

9. Kolo P, Sanya E, Ogunmodede J, Omotoso A, Soladoye A. Normotensive offspring of hypertensive Nigerians have increased Left ventricular mass and abnormal geometric patterns. Pan African Medical Journal. 2012;11(1). 